\title{
Immunologic aspects
}

\section{Immunity in dilated cardiomyopathy}

\author{
Sunil K. Das ${ }^{1}$, James T. Cassidy ${ }^{1}$, and Sally A. Huber ${ }^{2}$ \\ ${ }^{1}$ Department of Internal Medicine, The University of Michigan Medical School, Ann Arbor, Michigan, USA \\ ${ }^{2}$ Department of Pathology, The University of Vermont Medical College, Burlington, Vermont, USA
}

\begin{abstract}
Summary. Although several immunologic parameters have been found to be abnormal, their precise relationship to the cause of the disease remains unclear. It is believed that a virus initiating a bout of myocarditis may play a role in the perpetuation of the chronic phase, probably via immunologic mediation. A selection acquired defect or damage to a subpopulation of suppressor T lymphocytes is possible, providing the basis for the development of an autoimmune disorder. Studies in mice have shown a clear link between active viral myocarditis and the subsequent development of cardiomyopathy. However, the trigger mechanism that initiates the cardiomyopathy is still elusive. Specific antigens, possibly altered virus or damaged cardiac tissue, may be playing the role of the "hidden or cloistered" antigen in eliciting the immunologic reactions through a cytotoxic $T$ cell mechanism. Identification and isolation of the specific "antigen" in question could resolve the dilemmas about the immunopathogenesis of cardiomyopathy in humans.
\end{abstract}

The purpose of this communication is to review and examine the role of immunologic factors in dilated cardiomyopathy (DCM) in man. In this review we will focus on the role of (1) heart reactive antibodies, (2) cell-mediated immunity, and adduce (3) relevant data from experimental models of myocarditis that have bearing on the mechanisms involved in acute myocarditis; we will speculate on the possible link to subsequent development of dilated cardiomyopathy.

\section{Possible virus etiology in dilated cardiomyopathy}

Despite continuing present and past efforts, the etiology of dilated cardiomyopathy remains elusive. A nonspecific upper respiratory illness not infrequently heralds the onset of severe cardiac symptoms. In this setting, increased neutralizing antibody titers against Coxsackie B, echo and herpes virus have been noted by Sinani et al. [1] as well as by Kawai and Takutsu, [2]. Patients with other forms of heart disease did not show similar elevation in viral titers. Cambridge et al. have noted significantly higher viral titers in DCM, particularly in those with illness for less than 1 year, compared with age- and sex-matched patients with other forms of heart disease [3]. As additional support for the viral etiology, Kawai reported the presence of viral antigens in biopsy specimens of myocardium from a patient with DCM [4]. It is well recognized that only a few patients with infections due to the ubiquitous virus developed the characteristic syndrome alluded to above. Although genetic predisposition has not been systematically studied, Cambridge et al. found that HLA distribution frequencies in 20 of their patients with DCM were no different from those observed in the general population [3]. Not all patients with clinical myocarditis necessarily developed features of dilated cardiomyopathy at long-term follow-up [5]. Although the initial symptoms and signs in myocarditis may be attributed to viral invasion of the myocardium, the subsequent course of the disease, namely the chronic indolent form clinically recognized as dilated cardiomyopathy, is not thought to be due to persistence of viral effects on the myocardium [1]. At this chronic stage, an immune mediation is suspected, possibly triggered initially by the virus or perhaps by new antigens formed from necrotic myocardium and perpetuated via an autoimmune process [6]. Clinical support for incrimination of an immunologic mediation in DCM include: (1) the observation that cardiac symptoms follow an upper respiratory infection in previously healthy individuals, (2) a clinical course punctuated by recurrences of heart failure without obvious cause, (3) occasional clinical response to antiinflammatory drugs, (4) the demonstration of heart reactive antibiodies in some patients along with other serologic abnormalities, including hypergammaglobulinemia, antinuclear antibodies, rheumatoid factors, and biologic falsepositive serologic tests for syphilis, (5) demonstration of abnormality in cell-mediated immunity in various proportions of these patients, and (6) the not infrequent findings of lymphocytic and mononuclear infiltrates in the myocardium of these patients.

\section{Heart reactive antibodies}

The prevalence of heart reactive antibodies (HRA) in DCM has varied from $17 \%$ to $40 \%$. Because of their relative low prevalence and occurrence in other forms of heart disease, sometimes in higher frequency than in cardiomyopathy, doubt has been cast on whether these antibodies have any 
pathogenic role in the disease [7]. However, Bolte and Schultheiss showed that presence of HRA correlated with severity of symptoms and duration of the disease [8]. Previously we had observed that patients who were significantly disabled (class IV, New Heart Association) and who underwent cardiac transplantation had no circulating HRA prior to their transplant operation [9]. Despite the absence of HRA in the serum, cardiac tissue from these individuals showed extensive deposits of bound gammaglobulin and complement. It was postulated from this observation that the heart in advanced cardiomyopathy may preferentially fix heart reactive immunoglobulins to specific sarcolemmal and subsarcolemmal antigens, and consequently HRA may disappear from the serum. It was also surmised that binding of HRA may result in decreased ventricular function, either directly or indirectly via an inflammatory process. In a later study, Bolte, et al. correlated lgG binding to myocardial tissue with ejection fraction in patients with DCM [10]. Approximately $75 \%$ of patients with an ejection fraction of less than $35 \%$ showed $\operatorname{lgG}$ binding to myocardial tissue compared with approximately $45 \%$ of a second group of patients with DCM whose ejection fraction was greater than $35 \%$. In a miscellaneous group with cardiomyopathy due to various causes, including coronary artery disease and an ejection fraction greater than $50 \%$, lgG binding was found in only $25 \%$ of the patients. This study did not, however, evaluate similar patients with low ejection fraction. There is doubt as to the role HRA may play in the chronic phase of the disease as they are felt to be the result of injury regardless of the nature of the injury. However, in a limited study, Bengmark et al. showed that sera of patients with myocarditis, including two with idiopathic pericarditis, had cytotoxic effects on cardiac explants of newborn rat heart cells [11]. Technical difficulties, including the problems of obtaining and culturing adult heart cells and using them as target cells in cytotoxic experiments, thwarted extensive investigations of this type. Using newer techniques in cell preparation, Maisch et al. have shown that antisarcolemmal antibiodies, via antibody-dependent cytotoxicity, may play a role in the pathogenesis of human myocarditis [12]. In a very important follow-up study, these authors showed that all patients with DCM with prior evidence of myocarditis had antimyolemmal antibodies, which induce cytolysis of vital cardiocytes [13]. Furthermore, the titers of antibody correlated with the degree of cardiocytolysis. Thus, in the light of these studies, the whole issue about the role of HRA in myocarditis and DCM may have to be re-examined.

\section{Cell-mediated immunity in DCM}

Concurrent with the demonstration of HRA and DCM, simultaneous studies of cell-mediated immunity (CMI) were carried out. In our earlier study, CMI was evaluated by studying phytohemagglutinin (PHA) and human heart extract induced lymphocyte transformation [14]. Lymphocytes from 14 patients with cardiomyopathy, 17 with other forms of heart disease (five ischemic, eight rheumatic, three congenital, and one myocarditis), and 22 normal subjects were investigated. Transformation was expressed as a ratio of counts of tritiated thymidine uptake by lymphocytes stimulated by PHA or heart extract to unstimulated lymphocytes (S/u ratio). An S/u ratio equal to or greater than 50 indicated intact or normal CMI to mitogen, while an S/u ratio less than 10 indicated markedly depressed CMI. With heart extract, an $S / u$ ratio of less than 2 was normal, while one greater than 2 was considered abnormal, consistent with prior sensitization of the lymphocytes. The $\mathrm{S} / \mathrm{u}$ ratio with PHA for the cardiomyopathy group was $83 \pm 26$ (mean \pm SEM), for the heart disease group $93 \pm 17$, and for the normal control individuals $262 \pm 31$. The differences observed in the $\mathrm{S} / \mathrm{u}$ ratios between the normal and cardiomyopathy groups and between the normal and heart disease groups was significant $(P<0.01)$. A bimodal distribution of $\mathrm{S} / \mathrm{u}$ ratios was evident among the cardiomyopathy patients. Six in this group had an S/u ratio with PHA of less than 10 , indicating severely depressed CMI, whereas no patients in the disease control group or in the normal group had $\mathrm{S} / \mathrm{u}$ ratios this low. Clinical features, such as age, sex, duration and severity of disease, drugs used, and results of standard delayed hypersensitivity skin tests, were not different among the cardiomyopathy patients with low or normal PHA responses. Crossover studies using $\mathrm{AB}+$ in place of autologous serum resulted in a significant return toward normal in the PHA responses in these patients. Of interest is the observation that sera of three of the six patients prevented normal peripheral blood lymphocytes from responding to PHA. All three of these patients died from their disease soon afterward.

Lymphocyte responses to heart muscle showed that the $S / u$ ratio for the cardiomyopathy group was $1.9 \pm 0.5$, for the heart disease group $2.3 \pm 1$, and for the normal group $1.2 \pm 0.1$. The differences between each of these groups were not significant. An S/u ratio greater than 2 consistent with prior cardiac sensitization to heart muscle was seen in four patients with cardiomyopathy as well as in two with heart disease, both with rheumatic etiology, and one normal subject. This study suggests that cardiomyopathy does not represent a homogenous disorder from an immunologic standpoint as approximately $30 \%-40 \%$ of the patients showed evidence of abnormal CMI. Sachs and Lanfranchi showed reduced lymphocyte stimulation to PHA as well as absolute reduction in the percentage of circulating $T$ lymphocytes in a high proportion of their patients with cardiomyopathy [15]. Using the leukocyte migration inhibition test (LMI), another in vitro correlate of CMI, we investigated 12 patients with cardiomyopathy, 27 patients with ischemic heart disease (IHD), and 19 normal subjects [16]. Conconavalin $\mathrm{A}$ (Con $\mathrm{A}$ ) and human heart muscle extract as antigen were used in the two-stage LMI assay. Although the mean LMI responses to Con A were not significantly different in the three groups, a distinct bimodal response above and below a $90 \%$ confidence limit was noted in the DCM group. Five of twenty-seven patients with IHD $(19 \%), 2$ of 19 normal subjects ( $11 \%$ ), and 5 of 12 patients with DCM $(42 \%)$ had abnormal responses to Con A (above the $90 \%$ confidence limit). The proportion of patients with abnormal Con A responses was significantly higher in the DCM group than in the normal group $(P<0.05)$. Although LMI to heart extract was noted in 3 of 12 patients with DCM $(25 \%)$, 14 of 27 patients with IHD $(52 \%)$, and 2 of $19(10 \%)$ of the control group, there was a significant difference in the mean LMI response to heart extract only between the IHD group and the control group $(P<0.05)$. Again, this study, similar to the results from lymphocyte transformation cited earlier, indicated that only $40 \%$ of these patients may show dis- 
turbances of CMI. However, Moulias et al. found a higher proportion of their patients with DCM that exhibited abnormal LMI to myocardial extract [17].

In another type of study, Jacobs et al. studied CMI in a variety of heart diseases in order to assess its role in DCM [18]. They found that lymphocytes from 23 of 73 patients with DCM $(30 \%), 36$ of 148 patients with other forms of heart disease ( $24 \%$ ), mainly rheumatic and ischemic, and 2 of 49 normal subjects ( $4.9 \%$ ) showed evidence of cytotoxicity to cultured human heart cells. These authors concluded that lymphocyte cytotoxicity to heart muscle was not specific for DCM but may also occur following myocardial damage due to a variety of causes. Despite the technical limitations in performing this study, it represents an important attempt at elucidating the pathogenic mechanisms in DCM. Anderson et al. suggested another possible immune mechanism in DCM [19]. They noted that 6 of 37 patients with DCM who were under the age of 40 years and who had received cardiac transplants because of severe heart failure developed lymphoma, while 0 of 54 patients with coronary artery disease who received similar allografts developed malignancy. Serum from one of the patients with lymphoma inhibited normal suppressor cell function, while sera from the other five were not tested. Normally, suppressor $T$ cells prevent excessive lymphoid proliferation as a result of antigenic stimulation. It was postulated that in circumstances where suppressor $\mathrm{T}$-cell function was defective, antigenic stimulation to alloantigens may lead to abnormal lymphoid proliferation, sometimes resulting in lymphoma. Following up on this observation, Fowles et al. demonstrated defective T-suppressor cell function in DCM but not in coronary artery disease [20]. Eckstein et al. have shown similar decreased suppressor $T$-cell activity in patients with DCM and myocarditis [21]. However, in another study by Anderson et al. generalized defective suppressor $\mathrm{T}$-cell function was not confirmed [22]. Recently, defective natural killer cell activity has also been shown in about $50 \%$ of patients with DCM, raising an important question as to the pathogenesis of DCM [23]. Natural killer cells with non-T and non-B characteristics are known to provide early defense against invading virus infection. Defective killer cell activity could thus allow for the insidious propagation of the inflammatory process. The above authors also noted that HLA-A3 tissue type was associated with defective natural killer activity in their patients. Many immunologic processes are governed by a variety of cell-cell mechanisms regulated by the T-helper cells as well as by T-suppressor cells. Waldmann et al. have reviewed theories on the pathogenesis of immune deficiency and autoimmune diseases related to abnormal suppressor mechanisms [24]. Significant suppressor cell dysfunction has been elucidated as an important underlying mechanism in autoimmune diseases, such as systemic lupus erythematoses [25]. If suppressor cell function is indeed defective, future research may profitably be focused on investigating factors affecting its role, i.e., serum factors, acquired or inherited abnormalities, or destruction of these specific subsets of $\mathrm{T}$ cells.

\section{Experimental myocarditis}

Recent studies of experimental myocarditis have led to an important understanding in the pathogenesis of myocarditis and possibly in human DCM. In an important study, Wilson et al. showed that Coxsackie B virus-induced infection in mice resulted in active myocarditis, associated with viral replication in myocytes and recovery of virus from the myocardium for periods up to 1 week [26]. This phase was followed by a period of chronic indolent myocarditis during which virus was not recoverable. The histologic changes during this phase were not dissimilar to those encountered in human cardiomyopathy. An immune mechanism was thought likely as a possible cause for these late changes. In another important study, Matsumori and Kawai, for the first time, produced in experimental mice injected with encephalomyocarditis virus typical histologic features, along with cardiac dilatation, seen in human DCM long after the acute phase of myocarditis has resolved [27]. These changes were associated with increased mortality ascribed to congestive heart failure. Woodruff and Woodruff in their pioneering studies using Coxsackie-infected mice showed that prior $T$-cell deprivation led to significant reduction in the severity of the myocarditis and subsequent mortality [28]. Wong et al. from the same laboratory showed that infected animals could mount cell-mediated immune responses with the production of specific $\mathrm{T}$ cells, having cytotoxic properties directed particularly against in vitro infected cells [29]. However, these activated $T$ cells, if recovered from the spleen, did not show similar cytotoxicity against uninfected myocytes. Subsequently, Huber et al. confirmed the presence of $\mathrm{T}$ cytotoxic cells recovered from the spleen 5 days after the infection [30]. Furthermore, these authors showed that pretreatment of cytotoxic cells with antilymphocyte serum (anti-THY 1,2) and complement prevented lysing of the target cells. However, incubation with immune serum and complement did not abolish or reduce the cytotoxic potential of the cells. Maximal cytotoxicity occurred against infected syngeneic myofibers, as opposed to allogeneic myocytes, providing evidence that virus-specific effector cells were $T$ lymphocytes. These authors in a study showed that splenic cells which were recovered early ( 3 days after viral innoculation) could also cause lysis of uninfected myocytes [31]. The effector cells killed a wide range of target cells, including myocytes, endothelial cells, and fibroblasts. Their cytotoxicity was not inhibited by either anti-lg or anti-THY 1,2 antisera, unlike cytotoxic $\mathrm{T}$ cells recovered late $(5$ days after infection). It was postulated that the early effector cells might represent a population of natural killer cells. In this study, these authors, having noted previously that female mice infected with Coxsackie B3 virus developed a milder degree of inflammatory myocarditis and subsequent lower mortality than males, showed that infected female mice did not produce appreciable numbers of T-cytotoxic cells compared with the males. Female mice produced almost exclusively natural killer cell populations, which were less cytopathic than the other immune T-cytotoxic cells. Castration of the male mice prior to viral infection resulted in reduced mortality, myocardial inflammatory responses, and the development of T-cytotoxic lymphocytes [32].

Thus, in the course of experimental myocarditis, at least two sets of effector cell populations appear. The earlier set observed usually within 3 days of infection probably represents natural killer cells. These cells have nonspecific cytotoxic properties against a wide range of target cells. It is possible that these killer cells might serve to clear or contain the primary infection. The second set of effector cells with 
characteristics of $T$ cells is observed later, usually 5 days after infection, and they have specific effects directed toward the virus-infected or damaged myocytes with lysis of syngeneic target cells. It is envisioned that these T-cytotoxic or autoimmune cells might be partially responsible for the acute and possibly the chronic inflammatory changes observed in these models. Further studies with respect to the mechanism of the chronic changes in the myocardium leading to the stage of dilated cardiomyopathy are eagerly awaited.

\section{References}

1. Sinani GS, Krompotic E, Slodki SJ (1968) Adult heart disease due to Coxsackie virus $B$ infection. Medicine 47: 133-147

2. Kawai C, Takatsu T (1975) Clinical and experimental studies on cardiomyopathy. N Engl J Med 293: 529-597

3. Cambridge G, MacArthur CGC, Waterson AP, Goodwin JF, Oakley CM (1979) Antibodies to Coxsackie B viruses in congestive cardiomyopathy. Br Heart J 41:692-696

4. Kawai C (1971) Idiopathic cardiomyopathy. A study on the infectious immune theory as a cause of the disease. Jpn Circ J 35: $765-770$

5. Das SK, Brady TJ, Thrall JH, Pitt B (1980) Cardiac function in patients with prior myocarditis. J Nucl Med 21:689-693

6. Saunders V (1963) Viral myocarditis. Am Heart J 65: 707-713

7. Das SK, Cassidy JT, Petty RE (1974) The significance of heart reactive antibodies in heart disease. Chest 66:179-181

8. Bolte H-D, Schultheiss P (1978) Immunological results in myocardial diseases. Postgrad Med J 54: 500-503

9. Das SK, Callen IP, Dodson VN, Cassidy IT (1971) Immunoglobulin binding in cardiomyopathic hearts. Circulation 44: $612-616$

10. Bolte H-D, Schultheiss P, Cryen J, Goss F (1980) Binding of immunoglobulins in myocardium (biopsies) in cardiomyopathies. In: Bolte H-D (ed) Myocardial biopsy-Diagnostic significance. Springer, Berlin Heidelberg New York, pp 85-93

11. Bengmark S, Frisen L, Helander E (1963) A cytotoxic serum factor in heart disease, especially myocarditis. Acta Allergol 18: 479-488

12. Maisch B, Trostel-Soeder R, Berg PA, Kochsiek K (1981) Assessment of antibody mediated cytolysis of adult cardiocytes isolated by centrifugation in a continuous gradient of Percoll in patients with acute myocarditis. J Immunol Meth 44: 159-169

13. Maisch B, Deeg P, Liebau G, Kochsiek K (1983) Diagnostic relevance of human and cytotoxic immune reactions in primary and secondary dilated cardiomyopathy. Am J Cardiol 52: 1072-1078

14. Das SK, Petty RE, Meengs WL, Tubergen DC (1976) Cell mediated immunity in cardiomyopathy. Circulation $53 / 54$ (Suppl 2): 11-22

15. Sachs RN, Lanfranchi J (1978) Cardiomyopathies primitives et anomalies immunitaries. Cour Med Interne 17: 193-198

16. Das SK, Stein LD, Reynolds RT, Thebert P, Cassidy JT (1981) Immunologic studies in cardiomyopathy and pathophysiologic implications. Goodwin JF, Hjalmarson A, Olsen EGJ (eds). Lindgren \& Sonor, Molndal, Sweden, pp 87-93

17. Moulias R, Lafitte G, Komajda M, Buffet C, Congy F, Wang A (1978) Pathologie cardiaque et auto-immunite cellularie anticoeur. Cour Med Interne 17: 175-181

18. Jacobs B, Matsuda Y, Deodhar S, Shirey E (1979) Cell-mediated cytotoxicity to cardiac cells of lymphocytes from patients with primary myocardial disease. Am J Clin Pathol 72: 1-4

19. Anderson JL, Bieber CP, Fowles RE, Stinson EB (1978) Idiopathic cardiomyopathy, age, and suppressor-cell dysfunction as risk determinants of lymphoma after cardiac transplantation. Lancet II: 1174-1177

20. Fowles RE, Bieber CP, Stinson EB (1979) Defective in vitro suppressor cell function in idiopathic congestive cardiomyopathy. Circulation 59:483-491

21. Eckstein R, Mempel W, Bolte H-D (1982) Reduced suppressor cell activity in congestive cardiomyopathy and in myocarditis. Circulation 65: 1224-1229

22. Anderson JL, Greenwood JH, Kawaniski H (1981) Evaluation of suppressor immune regulatory function in idiopathic congestive cardiomyopathy and rheumatic heart disease. Br Heart $\mathrm{J}$ 46: $410-414$

23. Anderson JL, Carlquist JF, Hammond EH (1982) Deficient natural killer cell activity in patients with idiopathic dilated cardiomyopathy. Lancet II: 1124-1127

24. Waldmann TA, Blaese RM, Broder S, Krakauer RS (1978) Disorders of suppressor immunoregulatory cells in the pathogenesis of immunodeficiency and autoimmunity. Ann Intern Med 88: 226-238

25. Sagawa A, Abdou NI (1978) Suppressor-cell dysfunction in systemic lupus erythematosus. Cells involved and in vitro correction. J Clin Invest 62:789-796

26. Wilson FM, Miranda QR, Chason JL, Lerner AM (1969) Residual pathologic changes following murine Coxsackie $A$ and $B$ myocarditis. Am J Pathol 55:253-265

27. Matsumori A, Kawai $C$ (1982) An animal model of congestive (dilated) cardiomyopathy: dilatation and hypertrophy of the heart in the chronic stage in $\mathrm{DBA} / 2$ mice with myocarditis caused by encephalomyocarditis (EMC) virus. Circulation 66: $355-360$

28. Woodruff JF, Woodruff JJ (1974) Involvement of T lymphocytes in the pathogenesis of Coxsackie virus $B_{3}$ heart disease. $J$ Immunol 113:1726-1634

29. Wong CY, Woodruff JJ, Woodruff JF (1977) Generation of cytotoxic $T$ lymphocytes during Coxsackie virus B-3 infection. II. Characterization of effector cells and demonstration of cytotoxicity against viral-infected myofibers. J Immunol 118: 1165-1169

30. Huber SA, Job LP, Woodruff JF (1980) Lysis of infected myofibers by Coxsackic virus B-3-Immune T lymphocytes. Am J Path 98: 681-694

31. Huber SA, Job LP, Auld KR, Woodruff JF (1981) Sex related differences in the rapid production of cytotoxic spleen cells active against uninfected myofibers during Coxsackie virus B-3 infection. J Immunol 126: 1336-1340

32. Huber SA, Job LP, Auld KR (1982) Influence of sex hormones on Coxsackie B-3 virus infections in Balb/c mice. Cellular Immunol 67: 173-189

\section{Discussion}

\section{Discussants: Das, Maisch, Spry, Amorim, Kitaura}

It was concluded that there is evidence for an immunosuppressive factor or factors in dilated cardiomyopathy and that there is some experimental evidence of damage to myofibers caused by sensitized lymphocytes. 